\title{
Energy-Efficient Target Localization Based on a Prediction Model*
}

\author{
Yu Gu, Wei Zhang, HengChang Liu, Baohua Zhao, and Yugui Qu \\ Department of Computer Science, University of Science and Technology of China, \\ Hefei, Anhui 230027, China \\ Laboratory of Computer Science Institute of Software Chinese Academy of Sciences \\ \{guyu, , holiu\}@mail.ustc.edu.cn \\ \{bhzhao, ygqu\}@ustc.edu.cn
}

\begin{abstract}
In this paper, we propose an energy-efficient target localization scheme (TLS) based on a prediction model that can reveal the most likely zone the target will be in, and also a corresponding two-step communication protocol between base station (BS) and sensors. BS uses a query mechanism to determine which sensors should be used for detailed information according to a limited amount of data received from the sensors. This scheme reduces both energy consumption and communication bandwidth requirement, prolongs the lifetime of the wireless sensor networks. Simulation results indicate that it can achieve a high accuracy while saving a large amount of energy.
\end{abstract}

Keywords: wireless sensor networks, energy-efficient, prediction model, TLS.

\section{Introduction}

Many sensor networks need to handle physical entities that move in the environment (e.g. habitat monitoring [1] and intruder tracking [2]). Only sensors close to the target should participate in the aggregation of data associated with that target, since activating distant sensor nodes wastes precious energy without improving sensing fidelity. In recent research, a reliable mobicast method has been proposed to solve this kind of problem in literature [3] and achieved comparatively good results. Since energy management in the localization event is crucial for battery-driven sensor nodes where are severely energy constrained, here we focus on reducing energy consumption in wireless sensor networks for target localization.

In general, a sensor network has an almost constant rate of energy consumption if no target activities are detected in the sensor field. The minimization of energy consumption for an active sensor network with target activities is more complicated since target detection involves collaborative sensing and communication with different sensors.

* This paper is supported by the National Natural Science Foundation of China under Grant No. 60241004, the National Grand Fundamental Research 973 Program of China under Grant No. 2003CB314801, and the State Key Laboratory of Networking and Switching Technology. 
Though many literatures [4] [7] [8] [9] [11] have already introduced different methods to save energy during tracking procedure, without prediction knowledge of the target, most of energy consumed in the localization event still has been wasted in keeping too many sensors in the sensing state while actually only a few sensors will be enough.

To continuously monitor a mobile entity, a sensor network must maintain an active group that moves at the same velocity as the entity. This energy-efficient operation requires a prediction model to make conjectures about future target positions. Here in this paper, an energy-efficient target localization scheme (TLS) based on a prediction model has been posted. Since estimating the accurate future positions is almost impossible, the prediction model uses previous target position information to reveal the most likely zone (We call this zone as an awaken zone (AZ)) target will be in at the next moment, and then BS sends commands to wake up enough sensors inside AZ to track the target. We will show how to calculate AZ at time $\mathrm{i}$ and prove that there will always be some sensors in $\mathrm{AZ}$ that can detect the target under some assumptions. To the maximum of my knowledge, no previous work has been done on the exact prediction model of saving energy.

Two distinct improving results have been obtained:

- This scheme reduces averagely $80 \%$ energy consumption during the track procedure as the simulation shows.

- Analysis on TLS indicates that the requirement of communication bandwidth can also be reduced since the packets generated in the communication protocol are all kept very small.

In the past few years, a number of detecting and tracking methods have been proposed [4] [5] [6] [7] [8] [9] [10] [11] [12]. Those literatures focus either on how to reduce energy consumption or on how to localize a target more accurately.

Yi Zou and Krishnendu Chakrabarty have proposed an energy-aware target detection and localization strategy for cluster-based wireless networks in [4]. This strategy is based on a posteriori algorithm with a two-step communication protocol between cluster head and the sensor within the cluster. It uses a detection probability table which contains entries for all possible detection reports from those sensors that can detect a target at grid points to find out the target's max-probability position. In [7], T.Clouqueur and his copartners have discussed sensor deployment for collaborative target detection where path exposure is used as a measure of the effectiveness of the sensor deployment. Richard R. Brooks and his copartner have proposed a formulation which is anchored on location-aware data routing to conserve system resources, such as energy and bandwidth [9]. Literature [12] models the problem of a sensor network tracking a moving target as a Markov model of mobility, that is, the position of the target at time i depends only on its position at time i-1.

Range-based protocols use absolute point-to-point distance or angle information to calculate location between neighboring sensors. Common techniques for distance/angle estimation include Time of Arrival (TOA) [14], Time Difference of Arrival (TDOA) [13], Angle of Arrival (AOA) [15], and Received Signal Strength (RSS) [13]. Those protocols all can work well in our scheme. 
Considering that sensor density inside some AZ may be too high, we only need to wake up enough sensors in AZ instead of all sensors. Here we use the ASCENT method proposed in [16].

The reminder of the paper is organized as follows. Section 2 introduces system model and a few consumptions. Section 3 describes details about TLS. Section 4 shows energy consumption analysis. In section 5, we present simulation results and our practical considerations. Section 6 concludes the paper and outlines directions for future work.

\section{System Model and Assumptions}

In this section, we will introduce several assumptions in part $\mathrm{A}$. In part $\mathrm{B}$, a prediction model is described, and an exact definition of AZ is given with the existence of sensors inside that will detect the target in the future moment proven.

\section{A. Assumptions}

For a simple analysis, we make the following assumptions in this paper:

- All sensors remain stationary after deployment phase.

- Sensors are able to communicate with BS, while BS has the exact deployment information.

- BS is much more powerful in computation capability than sensors. BS is responsible for all calculation, while sensors are functioning mainly as data collecting devices.

- Considering a wireless sensor network with $\mathrm{N}$ sensors deployed averagely in dual space, we regard it reasonable to assume that there are $\mathrm{k}$ sensors $\operatorname{per} \pi r_{s}^{2}\left(k \geq 1, k \in Z^{+}\right), \quad\left(r_{s}\right.$ represents the detection range of sensors).

\section{B. Prediction Model}

In this part, we introduce the prediction model for target localization. A key contribution of this model is that it supplies BS with the information of an area where the target will be at the next moment. If the speed of a target moving in a wireless sensor network is lower than a threshold (We will show that the threshold in actual applications can be set high enough in Section 6), we can prove that there is at least one sensor inside that area which can detect the target.

To predict the target's position at time i, we should use the previous position information for target trace is always continuous in an actual environment.

Definition 1. $\vec{I}, \vec{I}$ is the target trace. $\vec{I}=\left(I_{0}\left(x_{0}, y_{0}\right), \ldots . ., I_{i}\left(x_{i}, y_{i}\right) \ldots ..\right)$, $I_{i}\left(x_{i}, y_{i}\right) \mathrm{i} \geq 0$ represents the target's position at time $\mathrm{i}$.

Given the target trace from time 0 to time i-1: $\vec{I}=\left(I_{0}\left(x_{0}, y_{0}\right), \ldots . ., I_{i-1}\left(x_{i-1}, y_{i-1}\right)\right)$, we model target's position at time $i(i \geq 1)$ following a two-dimensional Gaussian distribution, which is centered at the prediction point $\left(x_{\text {pre }-i}, y_{\text {pre }-i}\right)$. Namely, the 
mean of the Gaussian distribution $\mu$ equals $\left(x_{\text {pre-i }}, y_{\text {pre-i }}\right)$, and the probability distribution function (PDF) for the target's position at time $\mathrm{i}$ is the following:

$$
f(x, y)=\frac{1}{2 \pi \sigma^{2}} e^{\frac{\left(x-x_{\text {pre }-i}\right)^{2}+\left(y-y_{\text {pre }-i}\right)^{2}}{2 \sigma^{2}}}
$$

(Here $\sigma$ is the standard deviation). Without loss of generality, we assume that the standard deviation for all PDF is identical.

The prediction point $\left(x_{\text {pre }-i}, y_{\text {pre-i }}\right)$ represents the most possible position of the target at time i. It depends on the prior information of the target trace. Here we apply two different methods according to different situations in the practical applications.

I . Suppose the target walks along the line: $y=y_{i-1}+\left(x-x_{i-1}\right) \tan \theta$, then we can define the prediction point as: ( $\Delta t$ is the time interval between time $\mathrm{i}-1$ and i)

$$
\left\{\begin{array}{l}
x_{\text {pre }-i}=x_{i-1}+V_{i-1} \Delta t \cos \theta \\
y_{\text {pre- } i}=y_{i-1}+V_{i-1} \Delta t \sin \theta \quad\left(\mathrm{i} \geq 2 \quad \theta=\arctan \left(\frac{y_{i-1}-y_{i-2}}{x_{i-1}-x_{i-2}}\right)\right)
\end{array}\right.
$$

$V_{i-1}$ represents target's instantaneous speed at time i-1:

$$
V_{i-1}=\frac{\left|I_{i-1}-I_{i-2}\right|}{\Delta t} \quad \mathrm{i} \geq 2
$$

II . If we do not have much useful prior information of the target, which is common in actual environment, then the target position at time i-1 would be used as the prediction point:

$$
\left\{\begin{array}{l}
x_{\text {pre-i }}=x_{i-1} \\
y_{\text {pre-i }}=y_{i-1} \quad \mathrm{i} \geq 1
\end{array}\right.
$$

Since it is difficult to estimate future target position accurately, here instead of predicting the target position at time $i$, we will predict an area large enough in which the target will be by using (1).

According to (1), if the target trace $\vec{I}=\left(I_{0}\left(x_{0}, y_{0}\right), \ldots ., I_{i-1}\left(x_{i-1}, y_{i-1}\right)\right)$ is known, the probability that target will be in the round centered at prediction point $\left(x_{\text {pre }-i}, y_{\text {pre-i }}\right)$ with radius $\mathrm{R}$ that we can calculate using Theorem1 (introduced below) is greater than a given threshold $H(0 \leq H \leq 1)$. It means that we can calculate an area in which the target will be at time i with a probability greater than $H$.

Theorem 1. Given a threshold $H(0 \leq H \leq 1), \exists R \geq|\sigma| \sqrt{2 \ln 1 / 1-H}$, the probability that target will be in the round centered at prediction point $\left(x_{\text {pre }-i}, y_{\text {pre-i }}\right)$ with radius $\mathrm{R}$ at time $\mathrm{i}$ is larger than $\mathrm{H}$. 
Proof: See Appendix A.

We have mentioned before that BS would wake up enough sensors in an awaken zone (AZ) for future position detection, and an exact definition of $A Z$ is given below:

Definition 2. (Awaken Zone-i) Awaken Zone-i is a round centered at point $\left(x_{\text {pre }-i}, y_{\text {pre-i}}\right)$ with radius $\mathrm{R}=\sigma \sqrt{2 \ln 1 / 1-H}$. BS sends command to wake up enough sensors inside AS-i to keep sensing target. (We will use AZ instead of AZ-i in the following context)

Although the threshold $H$ cannot be preset in the paper, we still suggest that in practical applications $\mathrm{H}$ should be larger than $90 \%$ to make sure enough sensors in $\mathrm{AZ}$ can detect the target.

In the following part, we will approve the existence of at least one sensor in $\mathrm{AZ}$ we defined could detect the target at time $\mathrm{i}$.

Definition 3. $V_{H}$. $V_{H}$ is the maximum speed a target can have if it can be detected by sensors in AZ. $V_{H}=l_{\max } / \Delta t$, where $l_{\max }$ is the result of equations following: $\left(r_{s}\right.$ represents the detection range of sensors):

$$
\left\{\begin{array}{l}
S=r_{s}^{2} \theta_{1}-r_{s}^{2} \sin \theta_{1} \cos \theta_{1}+R^{2} \theta_{2}-R^{2} \sin \theta_{2} \cos \theta_{2} \\
\mathrm{R}=\sigma \sqrt{2 \ln 1 / 1-H} \\
\mathrm{~S} * \mathrm{k} / \pi \mathrm{r}_{\mathrm{s}}{ }^{2}=1 \\
\theta_{1}=\cos ^{-1}\left(\frac{r_{s}^{2}+l_{\text {max }}^{2}-R^{2}}{2 r_{s} l_{\max }}\right) \theta_{2}=\cos ^{-1}\left(\frac{R^{2}+l_{\max }^{2}-r_{s}^{2}}{2 R l_{\max }}\right)
\end{array}\right.
$$

Theorem 2. According to our pervious assumption: there are $\mathrm{k}$ sensors per $\pi r_{s}^{2}$ averagely. $\left(k \geq 1, k \in Z^{+}\right)$, if $V_{i} \leq V_{H}$, there will be at least one sensor inside Awakening Zone-i that can detect the target at time i.

Proof: See Appendix A

In this section, we have introduced our sensor nodes mode, prediction model and an exact definition of AZ has been given including how to calculate an AZ-i. We have also proven that the existence of at least one sensor inside the AZ-i can detect the target at time i under some assumptions which will be discussed in Section 6.

\section{Target Localization Scheme (TLS)}

First we will discuss the common flow about TLS in this section, and then we will show detailed information about data types used in the communication protocol in part A. Since we apply distributed algorithms in TLS, two different types of timer shown in 
part B are needed in the protocol. To save precious energy, after gathering draw date from sensors that have detected the target at current time, TLS uses a query mechanism to determine which sensors should be queried for further information which will be described in part $\mathrm{C}$.

\section{A. Data Types}

There are four different data types in our communication protocol.

AWK: when a sensor node in idle state receives an AWK packet, it wakes up, sets up a KEEPALIVE timer and keeps sensing for a constant time $\Delta t$. If it is already in sensing state, then the KEEPALIVE timer would be reset. An AWK packet is very small; actually, one bit will be enough.

RPT: when a sensor node detects an object, it generates a RPT packet indicating rest energy it has and the distance between them then sends it to BS.

QUY: BS sends a QUY packet to a sensor node, and waits for a DAT packet. A query message can also be encoded into a few bits.

DAT: When a sensor node receives a QUY packet, it sends a DAT packet to BS. A DAT packet includes detailed information about the target.

Since all the packets generated in our communication protocol are very small, in fact all the information can be encoded into only a few bits, TLS could reduce bandwidth requirement and the latency as well.

\section{B. KEEPALIVE Timer and WAIT Timer}

When a sensor in the idle state receives an AWK packet, it wakes up and set a KEEPALIVE timer. While the KEEPALIVE timer does not expire, it keeps sensing. But if a senor already in the sensing state receives an AWK packet, it would reset the timer and keep sensing.

After BS receives a RPT message for time i, it sets a WAIT timer which indicates how long BS should wait for all RPT messages. When WAIT timer expires, BS executes Query Mechanism to query sensors for detailed information. We can set $3 \Delta t / 4$ as its value.

\section{BS Query Mechanism}

To save energy, we only need to query $k_{q}(i)$ sensors for detailed information at time $\mathrm{i}$. When BS receives a REQ packet, if it is the first time to receive a detection report for time i, BS sets a WAIT timer. After the WAIT timer expires, BS considers that all sensor nodes that detect the target at time slot $\Delta t$ between time $\mathrm{i}-1$ and time $\mathrm{i}$ have been reported. Then it uses Query Mechanism to select sensors to query.

Suppose there are $k(i)\left(k(i) \geq k_{q}(i)\right)$ sensors which have sent $\mathrm{RPT}<d, E_{r}>$ packets to BS at time i, (d represents the distance between the senor and the target, $E_{r}$ represents rest energy of the sensor, $r_{s}$ represents the sensing range). We build the following equation: 


$$
F=\alpha \frac{E_{r}}{E}+(1-\alpha) \frac{d}{r_{s}} \quad 0 \leq \alpha \leq 1
$$

BS must query sensor nodes that have larger $\mathrm{F}$ for further detailed information. Obviously, to save precious energy, we must query the sensor nodes which have the most rest energy. But if we want to achieve a higher accuracy, we should query the sensor nodes which have a nearer distance to the target. So we build this equation considering both the energy and accuracy to decide which sensors to query.

In this section, we have described detailed information about our scheme, and in Section 5, we will discuss the advantages of TLS in energy consumption.

\section{Energy Consumption Analysis}

In this section, to show TLS`s impact on energy consumption, we will compare energy consumption between two different tracking schemes: Tracking an object in WSN without TLS and with TLS. A simplified sensor energy consumption model is used here as a metric for evaluating energy consumption. Suppose a sensor has three basic energy consumption types: sensing, active, idle, and these power values are $\varphi_{s}, \varphi_{a}, \varphi_{i}$.

Definition 4. $T_{r}$, if some sensor node detects the target, it needs to send a RPT packet $<d, E_{r}>$ to BS, $T_{r}$ represents the time needed to transfer this peculiar packet. Since usually the length of this packet is constant, we assume that $T_{r}$ is a constant value。

Definition 5. $T_{s}$, if some sensor node has received a QUY packet from BS, it needs to send BS a DAT packet. We define $T_{s}$ as the time the sensor used to receive this QUY packet plus the time it needs to transfer a DAT packet to BS. It is also reasonable to assume $T_{s}$ as a constant value.

\section{A. Energy Consumption Analysis for Tracking an Object in WSN Without TLS}

Without available prediction information, we must keep all sensor nodes in sensing state to make sure the target can be detected. Assume there are $k(i)$ sensors that can detect the target at time i, but only $k_{q}(i)\left(k_{q}(i) \leq k(i)\right)$ sensors would be queried by BS for detailed information.

In time slot $\Delta t$ between time $\mathrm{i}-1$ and $\mathrm{i}$ :

Number of sensors in sensing state: $\mathrm{N}$

Number of sensors that need to generate RPT packets: $k(i)$

Number of sensors that need to generate DAT packets: $k_{q}(i)$

We evaluate the energy consumption as following: 


$$
\begin{gathered}
E_{i}=N \varphi_{s} \Delta t+k_{q}(i) \varphi_{a} T_{s}+k(i) \varphi_{a} T_{r} \\
E=\sum_{i=0}^{t_{\text {end }}} E_{i}
\end{gathered}
$$

\section{B. Energy Consumption Analysis for Tracking an Object in WSN with TLS}

In time slot $\Delta t$ between time $\mathrm{i}-1$ and $\mathrm{i}$, considering that there are $k^{\prime}(i)\left(k^{\prime}(i) \leq k(i)\right)$ sensors that can detect the target, but only $k_{q}(i)\left(k_{q}(i) \leq k(i)\right)$ sensors would be queried by BS for detailed information.

Number of sensors in the sensing state: $k\left(\frac{\sigma}{r_{s}}\right)^{2} \ln \frac{1}{1-H}$

Number of sensors that detect the target: $k^{\prime}(i) \quad\left(k^{\prime}(i) \leq k(i)\right)$

Number of sensors that need to generate DAT packets: $k_{q}(i)$

Number of sensors in the idle state: $N-k\left(\frac{\sigma}{r_{s}}\right)^{2} \ln \frac{1}{1-H}$

Energy consumption using TLS can be expressed as

$$
\begin{aligned}
E_{i} & =k\left(\frac{\sigma}{r_{s}}\right)^{2} \ln \frac{1}{1-H} \varphi_{s} \Delta t+k_{q}(i) \varphi_{a} T_{s}+k^{\prime}(i) \varphi_{a} T_{r}+\left(N-k\left(\frac{\sigma}{r_{s}}\right)^{2} \ln \frac{1}{1-H}\right) \varphi_{i} \Delta t \\
E & =\sum_{i=0}^{t_{\text {ond }}} E_{i}
\end{aligned}
$$

Therefore, the difference consumption $\Delta E_{i}=E_{i}-E_{i}$ can be expressed as:

$$
\begin{aligned}
\Delta E_{i} & =\left(N-k\left(\frac{\sigma}{r_{s}}\right)^{2} \ln \frac{1}{1-H}\right) \varphi_{s} \Delta t+\left(k(i)-k^{\prime}(i)\right) \varphi_{a} T_{r}-\left(N-k\left(\frac{\sigma}{r_{s}}\right)^{2} \ln \frac{1}{1-H}\right) \varphi_{i} \Delta t \\
& =\left(N-k\left(\frac{\sigma}{r_{s}}\right)^{2} \ln \frac{1}{1-H}\right)\left(\varphi_{s}-\varphi_{i}\right) \Delta t+\left(k(i)-k^{\prime}(i)\right) \varphi_{a} T_{r} \\
\Delta E & =\sum_{i=0}^{t_{\text {ond }}} \Delta E_{i}
\end{aligned}
$$

Since $\varphi_{s} \prec \varphi_{i} \& k(i) \geq k^{\prime}(i)$ (Because AZ is a part of the sensor field), we can see that the energy consumption is greatly reduced with the passage of time.

The comparison between tracking flows with TLS and without TLS has been shown in this section. We can see that the energy consumption is greatly reduced through equation (9) if using TLS. In the next section, we will show simulation results and practical consideration.

\section{Simulation and Practical Consideration}

We present results for a case study carried out using MatLab6.1 and C++ in this section. The simulation is done on a $100 \mathrm{~m}$ by $100 \mathrm{~m}$ field grid with 20 sensors randomly placed in the sensor field. 
The parameters of the prediction models are $\sigma=10, \Delta t=0.3 \mathrm{~s}, H=90 \%$, $r_{s}=15 \mathrm{~m}$. We choose the energy consumption model parameters as $\varphi_{a}=400 \mathrm{nj} / \mathrm{s}, \varphi_{i}=100 \mathrm{nj} / \mathrm{s}$ and $\varphi_{s}=1000 \mathrm{nj} / \mathrm{s}$, we have no physical data available for $T_{r}$ and $T_{s}$; however, their values do not affect the target localization procedure, therefore we only need to set them manually to satisfy the relationship $T_{r} \prec T_{s}$. In this case, $T_{r}=10 \mathrm{~ms}, T_{s}=100 \mathrm{~ms}$.

The layout of the sensor field is given in Fig 2, with a target trace randomly generated in the sensor field. The target travels from the position $(0,0)$. We assume the target locations are updated at discrete time instants in unit of seconds, and the granularity of the time is long enough for sampling by two neighboring locations in the target trace with negligible errors. For simplicity, we have evaluated TLS for $k_{q}(i)=1$. Fig 3 presents the instantaneous energy saving in percentage, and Fig 4 presents the energy consumption for the case study as the target moves along its trace in the sensor field. From Fig 3 and Fig 4, we note that a large amount of energy is saved during target localization. Averagely $80 \%$ energy could be saved if TLS has been used.

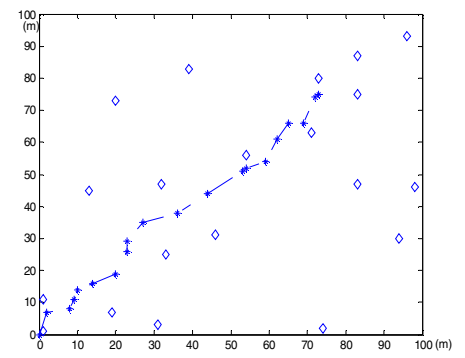

Fig. 1. Sensor Field with Target Trace

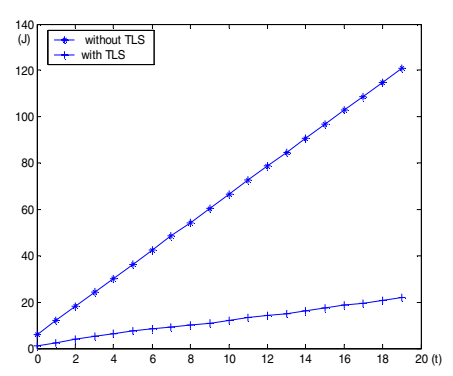

Fig. 3. Energy Consumption Comparison

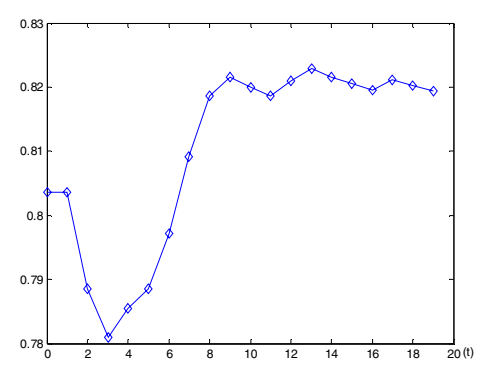

Fig. 2. Instantaneous Energy Saving in percentage

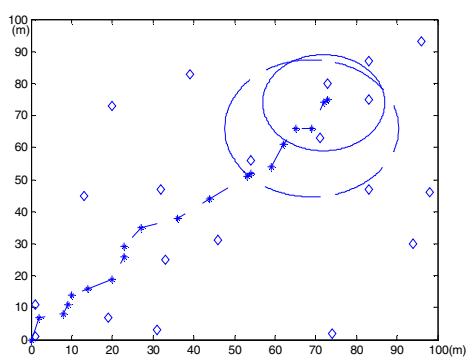

Fig. 4. Prediction Model Analysis

Fig 5 shows how our prediction model works: there are four sensors needed to be woken up inside the dashed round that presents the AZ-19, while actually three of them 
which are in the smaller round can detect the target at time 19. In our simulations, the prediction model has worked efficiently. (Here we use (4) to generate prediction points).

Practical consideration: In actual environment, according to our definition 3, we know that $V_{H}$ follows:

$$
\frac{R}{\Delta t}<V_{H}<\frac{R+r_{s}}{\Delta t}
$$

Let us use some reasonable, common data to check $V_{H}$ 's lower limit and upper limit, e.g: $\mathrm{H}=0.9$

$$
\begin{aligned}
\sigma=10 & \Rightarrow R=21 \mathrm{~m}, \Delta t=0.3 \mathrm{~s}, r_{s}=40 \mathrm{~m} \\
& \Rightarrow 70 \mathrm{~m} / \mathrm{s}<V_{H}<200 \mathrm{~m} / \mathrm{s} \\
& \Rightarrow 252 \mathrm{~km} / \mathrm{h}<V_{H}<720 \mathrm{~km} / \mathrm{h}
\end{aligned}
$$

We can see that $V_{H}$ is large enough for the target we track in the actual applications.

The standard deviation $\sigma$ in (1) can be gained using transcendental information about the target in actual applications, and we believe that $\sigma$ is proportion to $V_{H}$.

The simulation results shown in this section indicate that TLS runs smoothly in the tracking procedure while reducing energy consumption by $80 \%$.

\section{Conclusion and Future Work}

In this paper we bring forward an energy-efficient target localization scheme named TLS based on a prediction model for wireless sensor networks. The TLS make use of previous target position information and PDF knowledge of the target's trace to reveal which area the target will be in at the next moment, then a limited amount of sleeping sensors inside that area will be woke up to keep alert. We also propose a corresponding two-step communication protocol between sensors and BS. The analysis shows that TLS can effectively reduce energy consumption as the simulation results indicate. Since packets transferred in the procedure are kept very small, TLS decreases the latency for target localization as well.

In our future work, we will focus on improving the accuracy of the TLS. If the track knowledge mode we use cannot accurately model the actual track, there will be extra errors in the localization. We will study the properties of this kind of errors in our future work and try to propose an error-smooth method.

\section{References}

[1] A.Cerpa, J. Elson, D.Estrin, L.Girod, M.Hamilton, and J.Zhao "Habitat monitoring: Application driver for wireless communications technology" in ACM SIGCOMM Workshop on Data Communications in Latin America and the Caribbean, Costa Rica, April 2001.

[2] D.Li, K Wong, YH.Hu, and A.Sayeed "Detection, classification and tracking of targets in distributed sensor network” IEEE Signal Processing Magazine, Vol. 19, no. 2, March 2002. 
[3] Qingfeng Huang, Chenyang Lu and Gruia-Catalin Roman "Reliable Mobicast via Face-Aware Routing" In Proceedings of the 23rd Annual Joint Conference of the IEEE Computer and Communications Societies (INFOCOM 2004), March 2004.

[4] Yi Zou and Krishnendu Chakrabarty "Energy-Aware Target Localization in Wireless Sensor Networks" Proc IEEE International Conference on Pervasive Computing and Communications (PerCom 2003) pp. 60-67, 2003.

[5] Dragoş Niculescu "Positioning in Ad Hoc Sensor Networks" IEEE Network Magazine, July/August 2004. pp. 24-29.

[6] Hussam Al-Hertani and Jacek Ilow "Detection and Localizaition in a Wireless network of Randomly Distributed Sensors" CCGEI 2003, Montreal, May 2003.

[7] T.Clouqueur, V.Phipatanasuphorn, P.Ramanathan, and K.K.Saluja "Sensor Deployment Strategy for Target Detection", Proc. 1st ASM International Workshop on Wireless Sensor Networks and Applications, pp.42-48, September 2002.

[8] J.Liu, P.Cheung, L.Guibas, and F.Zhao "A Dual-Space Approach to Tracking and Sensor Management in Wireless Sensor Networks" Proc. 1st ACM International Workshop on Wireless Sensor Networks and Applications, pp. 131-139, September 2002.

[9] Richard R.Brooks, Parameswaran Ramanathan and Akbar M. Syeed "Distributed Target Classification and Tracking in Sensor Networks" Proceeding of the IEEE, Vol.91, No.8.August 2003

[10] Ruixin Niu and Pramod K.Varshney "Target Location Estimation in Wireless Senor Networks Using Binary Data" Proceedings of the 38th Annual Conference on Information Sciences and Systems, Princeton, NJ, March 2004.

[11] Xinbo Yu, Koushik Niyogi, Sharad Mehrotra and Nalini Venkatasubramanian "Adaptive Target Tracking in Sensor Networks" Communication Networks and Distributed Systems Modeling and Simulation conference (CNDS 04), 2004 San Diego.

[12] Jean-Paul Wagner and Razvan Cristescu "Power Control for Tracking in Sensor Networks" 2005 Conference On information Sciences and System, The Johns Hopkins university, March 16-18, 2005.

[13] P.Bahl and V.N.Padmanabhan "RADAR: An in-building RF-based user location and tracking system" In Proceeding of the IEEE INFOCOM, pages 775-784, March 2000.

[14] B.Hofman-Wellenhof, H.Lichtenegger, and J.Collins. "Global Positioning System: Theory and Practice" Springer Verlag, $4^{\text {th }}$ ed, 1997.

[15] D.Niculescu and B.Nath "Ad hoc positioning system (APS) using AoA" In Proceeding of IEEE INFOCOM 2003, pages 1734-1743, April 2003.

[16] Alberto Cerpa, Deborah Estrin "ASCENT: Adaptive Self-Configuring Sensor Networks Topologies" IEEE transactions on Mobile Computing, vol.3, no.3, July-September 2004.

\section{Appendix}

\section{A. Proof of Theorem 1 and 2}

Theorem 1. Given a threshold $H(0 \leq H \leq 1), \exists R \geq|\sigma| \sqrt{2 \ln 1 / 1-H}$, the probability that target will be in the round centered at prediction point $\left(x_{\text {pre-i }}, y_{\text {pre-i }}\right)$ with radius $\mathrm{R}$ at time $\mathrm{i}$ is greater than $\mathrm{H}$. 
Proof: Assume that $P(x, y)$ is the probability that the target will be in the round centered at prediction point $\left(x_{p r e-i}, y_{\text {pre }-i}\right)$ with radius $\mathrm{R}$ at time $\mathrm{i}$, according to (1): $P(x, y)=\underset{\left(\mathrm{x}-\mathrm{x}_{\mathrm{pre}-\mathrm{i}}\right)^{2}+\left(\mathrm{y}-\mathrm{y}_{\mathrm{pre}-\mathrm{i}}\right)^{2} \leq \mathrm{R}^{2}}{\int} f(x, y) d x d y$. Given a threshold $H(0 \leq H \leq 1)$, if $P(x, y) \geq H$, which means that: $\iint_{\left(\mathrm{x}-\mathrm{x}_{\mathrm{pre}-\mathrm{i}}\right)^{2}+\left(\mathrm{y}-\mathrm{y}_{\mathrm{pre}-\mathrm{i}}\right)^{2} \leq \mathrm{R}^{2}} f(x, y) d x d y \geq H$, let $\mathrm{u}=\frac{\mathbf{x}-\mathbf{x}_{\mathrm{pr}-\mathrm{i}}}{\sigma}$, $v=\frac{y-y_{p r e-i}}{\sigma}$

$\Rightarrow \frac{1}{2 \pi} \iint_{u^{2}+v^{2} \leq r_{0}^{2}} e^{-\frac{u^{2}+v^{2}}{2}} d u d v \geq H \Rightarrow r_{0} \geq \sqrt{2 \ln 1 / 1-H} \Rightarrow R \geq|\sigma| \sqrt{2 \ln 1 / 1-H}$

Theorem 2. According to our pervious assumption: there are $\mathrm{k}$ sensors per $\pi r_{s}^{2}$ averagely. $\left(k \geq 1, k \in Z^{+}\right)$, if $V_{i} \leq V_{H}$, there will be at least one sensor inside Awakening Zone-i that can detect target at time $i$.

Proof: According to Figure 2, if there is a sensor that can detect the target, it is inside the shadow $\mathrm{S}$ :

$$
\begin{array}{r}
S=r_{s}^{2} \theta_{1}-r_{s}^{2} \sin \theta_{1} \cos \theta_{1}+R^{2} \theta_{2}-R^{2} \sin \theta_{2} \cos \theta_{2} \\
\theta_{1}=\cos ^{-1}\left(\frac{r_{s}^{2}+l^{2}-R^{2}}{2 r_{s} l}\right) \theta_{2}=\cos ^{-1}\left(\frac{R^{2}+l^{2}-r_{s}^{2}}{2 R l}\right)
\end{array}
$$

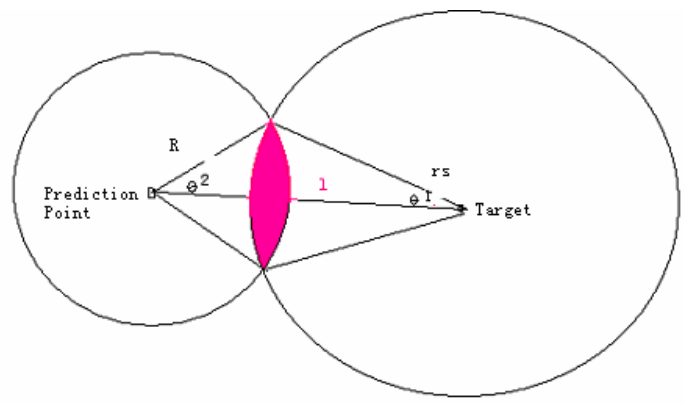

Fig. 1. An Example of $A Z$ and the Target

Since there are $\mathrm{k}$ sensors per $\pi r_{s}^{2}$ averagely, it is easy to know: $S * k / \pi r_{s}{ }^{2} \geq 1$. According to Definition 3, $V_{H}$ is the result of equation (6) when $S * k / \pi r_{s}^{2}=1$. So if $V_{i} \leq V_{H} \quad, S^{*} k / \pi r_{s}^{2} \geq 1$ which means that at least one sensor can detects the target. 


\section{B. Explanations of Variables in Paper}

Table 1. Explanations of variables appeared in the paper

\begin{tabular}{|c|c|c|c|}
\hline$I_{i}\left(x_{i}, y_{i}\right)$ & $\begin{array}{l}\text { Target's } \\
\text { position we } \\
\text { detect at time i. }\end{array}$ & $r_{s}$ & $\begin{array}{l}\text { Detection range of } \\
\text { sensors }\end{array}$ \\
\hline $\mathrm{k}$ & $\begin{array}{l}\text { Density of } \\
\text { WSN }\end{array}$ & $\varphi_{a}$ & $\begin{array}{l}\text { Power value of a } \\
\text { sensor in active state }\end{array}$ \\
\hline$\varphi_{s}$ & $\begin{array}{l}\text { Power value of } \\
\text { a sensor in } \\
\text { sensing state }\end{array}$ & $\varphi_{i}$ & $\begin{array}{l}\text { Power value of a } \\
\text { sensor in idle state }\end{array}$ \\
\hline$\left(x_{\text {pre- }-i}, y_{\text {pre- } i}\right)$ & $\begin{array}{l}\text { Prediction } \\
\text { position of the } \\
\text { target at time } i\end{array}$ & $V_{i-1}$ & $\begin{array}{l}\text { target's instantaneous } \\
\text { speed at time i-1: }\end{array}$ \\
\hline$\sigma$ & $\begin{array}{l}\text { The standard } \\
\text { deviation }\end{array}$ & $H(0 \leq H \leq 1)$ & threshold \\
\hline$R=|\sigma| \sqrt{2 \ln 1 / 1-H}$ & $\begin{array}{l}\text { The radius of } \\
\text { AZ }\end{array}$ & $l_{\max }$ & $l_{\max }=V_{H} * \Delta t$ \\
\hline$V_{H}$ & $\begin{array}{l}\text { The maximum } \\
\text { speed } t\end{array}$ & $\mathrm{~d}$ & $\begin{array}{l}\text { Distance between the } \\
\text { sensor and the target }\end{array}$ \\
\hline$E_{r}$ & Rest energy & $\alpha$ & $\begin{array}{l}\text { The percentage } \\
\text { variable }\end{array}$ \\
\hline $\mathrm{F}$ & $\begin{array}{l}\text { decides } \\
\text { whether the } \\
\text { sensor would } \\
\text { be queried by } \\
\text { BS }\end{array}$ & $k_{q}(i)$ & $\begin{array}{l}\text { Number of sensors } \\
\text { queried by BS at time } \\
\mathrm{i}\end{array}$ \\
\hline$k(i)$ & $\begin{array}{l}\text { Number of } \\
\text { sensors can } \\
\text { detect the } \\
\text { target at time i } \\
\text { without TLS }\end{array}$ & $k^{\prime}(i)$ & $\begin{array}{l}\text { Number of sensors } \\
\text { can detect the target at } \\
\text { time i with TLS }\end{array}$ \\
\hline$\Delta t$ & $\begin{array}{l}\text { Time interval } \\
\text { between time } \\
\mathrm{i}-1 \text { and } \mathrm{i}\end{array}$ & $E_{i}$ & $\begin{array}{l}\text { Energy consumption } \\
\text { between time } \mathrm{i}-1 \text { and } \mathrm{i} \\
\text { without TLS }\end{array}$ \\
\hline$E_{i}^{\prime}$ & $\begin{array}{l}\text { Energy } \\
\text { consumption } \\
\text { between time } \\
\mathrm{i}-1 \text { and i with } \\
\text { TLS }\end{array}$ & $\Delta E_{i}$ & $\begin{array}{ll}\text { Difference } & \text { between } \\
E_{i} \text { and } E_{i} & \end{array}$ \\
\hline
\end{tabular}

